\title{
Best cylindrical map projections according to the undesirability of angular and areal distortions
}

\author{
Kerkovits Krisztián* \\ Institute of Cartography and Geoinformatics, ELTE Eötvös Loránd University, Budapest, Hungary, kerkovits@map.elte.hu \\ * Corresponding Author
}

\begin{abstract}
Seeking low distortion maps, it is usual to assume that areal and angular distortions are equally undesirable on the map. However, this might not be the case for certain map thematics. Should angular distortions be a bit less preferred to areal distortions, maps of unbalanced distortions may be developed. In this paper, the known analytic solution for the best cylindrical map projection is extended to such more general requirements by utilizing calculus of variations. The overall distortion of the resulted mappings are calculated and compared to each other to explore the distortion characteristics of these intentionally unbalanced map projections.
\end{abstract}

Keywords: low-distortion map projections, cylindrical mappings, calculus of variations

\section{Introduction}

A map projection is cylindrical if it maps parallel lines to parallel straight lines and meridians to proportionally spaced straight lines that are orthogonal to the images of parallels (Lapaine and Frančula, 2016). Such maps are rarely used for printed small-scale maps due to their unfavourable distortion patterns but they might be useful for certain maps (e.g. depicting time zones). On the other hand these mappings are popular in web cartography: their obvious advantages are that they can completely fill the rectangular map frame and their straight antimeridian representation makes it possible to repeat the map continuously at the left and right edges eliminating the cuts at the antimeridian.

Among cylindrical mappings there are conformal or equalarea variants. If one would like to reduce both areal and angular distortions at the same time, the equidistant cylindrical mapping would be the best choice (Györffy, 1990). All three variants may have two distortion-free parallels symmetrical to the Equator, the optimal choice (resulting in the least distortion possible) was observed to be the same for all three variants (Frančula, 1971; Grafarend and Niermann, 1984).

While these solutions are well-known if areal and angular distortions are equally undesired, one may try to avoid areal distortion more than angular distortion. Former studies all optimized maps using distortion criteria that aimed to balance all kinds of distortions, but the cartographer may want to fine-tune the ratio of areal and angular distortions if he would like to avoid one of them more than the other.

In this paper, these results will be extended: the formula of the cylindrical map projections with the least distortion possible will be developed even if areal and angular distortions are not equally undesired. The optimal value of the standard parallel will also be calculated: its value will be independent from the relative undesirability of areal and angular distortions.
Kerkovits (2019) demonstrated that distortion of length strongly correlates to the linear combination of areal and angular distortions. Furthermore, Györffy (2016) also revealed a functional relationship between them. Thus, explicit inclusion of linear distortion may safely be omitted, as angular and areal distortions already count for its effect implicitly.

An exploratory study will be conducted on the resulting mappings: Their distortion values will be compared to each other to discover the effect of unequal undesirability in areal and angular distortions to the overall distortion value. This will show a symmetrical behaviour of areal and angular distortions on cylindrical mappings.

\section{Measuring distortion in cylindrical mappings}

As this study deals with small-scale maps, the difference between the ellipsoid of revolution and the sphere is negligible. Thus, the reference frame is considered as a sphere of unit radius parametrized by the latitude $\varphi$ and longitude $\lambda$. The planar Cartesian system is used for describing the map coordinates using $x$ for the horizontal and $y$ for the vertical axis. Angles in the formulae should be interpreted in radians and the map is assumed to be in unit scale. Using this notation, the general formulae of the cylindrical map projections are:

$$
\begin{gathered}
x=\lambda \cos \varphi_{s} \\
y=y(\varphi)
\end{gathered}
$$

where $y(\varphi)$ is a differentiable strictly increasing odd function and $\pm \varphi_{s}$ are the true-scale latitudes. They do not have to be necessarily standard parallels (i.e., distortion-free), only their length corresponds to the original spherical arc length.

Linear scale $h$ along meridians and $k$ along parallels are 
(Snyder, 1987):

$$
\begin{gathered}
h=\frac{\mathrm{d} y}{\mathrm{~d} \varphi}=y^{\prime} \\
k=\frac{\mathrm{d} x}{\mathrm{~d} \lambda} \frac{1}{\cos \varphi}=\frac{\cos \varphi_{s}}{\cos \varphi}
\end{gathered}
$$

Parallels and meridians are principal directions in all projections with rectangular graticule. Therefore, $h$ and $k$ can be substituted for minimal and maximal linear scales in the formulae.

The undesirability of areal and angular distortions may be unequal depending on the purpose of the map. Let us introduce the variable $0 \leq q \leq 1$ to express the undesirability of areal distortion compared to angular distortion. $q=1$ means that our map may not distort areas at all, $q=1 / 2$ denotes that areal and angular distortions are equally undesired, and $q=0$ stands for the need of a conformal map. The choice of $q \approx 2 / 3$ is notable, as the linear distortions of the map depend on areal distortions approximately twice as on angular distortions (Kerkovits, 2019), so this is the correct choice if one would like to present mapped distances with the least distortion possible.

To weight the areal and angular distortions correctly, one must use such formulae for their computation that measure them on a comparable scale. Баева [Bayeva] (1987) indicated that logarithmic formulae are apt for this purpose and this was confirmed also by Kerkovits (2020). The areal distortion value $\varepsilon_{p}^{2}$ and angular distortion value $\varepsilon_{i}^{2}$ is calculated as:

$$
\begin{gathered}
\varepsilon_{p}^{2}=\ln ^{2}(h k) \\
\varepsilon_{i}^{2}=\ln ^{2} \frac{h}{k}
\end{gathered}
$$

N. B. $\ln ^{2}(h / k)=\ln ^{2}(k / h)$, so no precautions are necessary to ensure that $h$ is the maximal rather than the minimal linear scale. The overall map distortion $E$ over a spherical surface $S$ can be measured effectively as the second moment (quadratic mean) of the local distortions, also known as the Airy-Kavrayskiy criterion (Frančula, 1971; Györffy, 2016; Kerkovits, 2020). Here, areal and angular distortions will be weighted with their undesirability $q$ and $1-q$ respectively:

$$
E^{2}=\frac{1}{S} \int_{S} q \varepsilon_{p}^{2}+(1-q) \varepsilon_{i}^{2} \mathrm{~d} S
$$

The theorem of Chebyshev states that the best conformal mapping has a constant distortion along the boundary of the mapped area and some kind of similar behaviour is observed on other kinds of map projections (Meshcheryakov, 1968). As cylindrical mappings have constant distortion along parallels, they are a good choice for spherical zones symmetrical to the Equator. Therefore, the surface $S$ will be a spherical zone bounded by latitudes $\pm \varphi_{B}$. The former equation becomes:

$$
\begin{aligned}
E^{2}=\frac{1}{2 \sin \varphi_{B}} \int_{-\varphi_{B}}^{\varphi_{B}}\left[q \ln ^{2} \frac{y^{\prime} \cos \varphi_{s}}{\cos \varphi}+\right. \\
\left.\quad(1-q) \ln ^{2} \frac{y^{\prime} \cos \varphi}{\cos \varphi_{s}}\right] \cos \varphi \mathrm{d} \varphi
\end{aligned}
$$

Note that the change of the integral variable necessitates the multiplication of the original integrand by $\cos \varphi$.

\section{Development of the best cylindrical mappings}

Equation (8) has the form of:

$$
E^{2}=\text { const. } \cdot \int_{-\varphi_{B}}^{\varphi_{B}} f\left(y^{\prime}, \varphi\right) \mathrm{d} \varphi
$$

We seek the minimum of this expression. A necessary condition for an extremal value of such an expression is to fulfil the Euler-Lagrange differential equation:

$$
\frac{\partial f}{\partial y}-\frac{\mathrm{d}}{\mathrm{d} \varphi}\left(\frac{\partial f}{\partial y^{\prime}}\right)=0
$$

In our case, $\partial f / \partial y=0$, which simplifies the former equation to $\partial f / \partial y^{\prime}=$ const . Another necessary condition of the minimum is the transversality condition:

$$
\left.\frac{\partial f}{\partial y^{\prime}}\right|_{\varphi= \pm \varphi_{B}}=0
$$

The two conditions together:

$$
\frac{\partial f}{\partial y^{\prime}} \equiv 0
$$

That is:

$$
\cos \varphi\left[\frac{2 q}{y^{\prime}} \ln \frac{y^{\prime} \cos \varphi_{s}}{\cos \varphi}+\frac{2(1-q)}{y^{\prime}} \ln \frac{y^{\prime} \cos \varphi}{\cos \varphi_{s}}\right]=0
$$

Solving for $\ln y^{\prime}$ :

$$
\begin{gathered}
(2 q-1) \ln \cos \varphi_{s}+\ln y^{\prime}+(1-2 q) \ln \cos \varphi=0 \\
\ln y^{\prime}=(1-2 q) \ln \frac{\cos \varphi_{s}}{\cos \varphi}
\end{gathered}
$$

Substituting (15) into (8):

$$
\begin{gathered}
E^{2}=\frac{1}{2 \sin \varphi_{B}} \int_{-\varphi_{B}}^{\varphi_{B}}\left[4 q(1-q)^{2} \ln ^{2} \frac{\cos \varphi_{s}}{\cos \varphi}+\right. \\
\left.4 q^{2}(1-q) \ln ^{2} \frac{\cos \varphi_{s}}{\cos \varphi}\right] \cos \varphi \mathrm{d} \varphi= \\
\frac{4 q(1-q)}{2 \sin \varphi_{B}} \int_{-\varphi_{B}}^{\varphi_{B}} \cos \varphi \ln ^{2} \frac{\cos \varphi_{s}}{\cos \varphi} \mathrm{d} \varphi
\end{gathered}
$$


Another necessary condition for the minimum of $E$ is:

$$
\frac{\partial E^{2}}{\partial \varphi_{s}}=0
$$

Using the Leibniz integral rule for the derivative of the integral:

$$
\begin{gathered}
\frac{2 q(1-q)}{\sin \varphi_{B}} \int_{-\varphi_{B}}^{\varphi_{B}} 2 \sin \varphi_{s} \frac{\cos \varphi}{\cos \varphi_{s}} \ln \frac{\cos \varphi_{s}}{\cos \varphi} \mathrm{d} \varphi=0 \\
\tan \varphi_{S} \int_{-\varphi_{B}}^{\varphi_{B}} \cos \varphi \ln \frac{\cos \varphi_{s}}{\cos \varphi} \mathrm{d} \varphi=0
\end{gathered}
$$

This has two distinct solutions. $\varphi_{s}=0$ is an extraneous root because it is at a local maximum of $E^{2}$. The other root, which is the global minimum of $E^{2}$ may be gained by evaluating the integral using the fundamental theorem of calculus:

$$
\ln \cos \varphi_{S}=\ln \cos \varphi_{B}-1+\frac{\ln \tan \left(\frac{\pi}{4}+\frac{\varphi_{B}}{2}\right)}{\sin \varphi_{B}}
$$

To uniquely define the cylindrical mapping, not only $\varphi_{s}$ is required but also $y$. However, from formula (15):

$$
\begin{gathered}
y^{\prime}=\left(\frac{\cos \varphi}{\cos \varphi_{s}}\right)^{2 q-1} \\
y=\cos ^{1-2 q} \varphi_{s} \int_{0}^{\varphi} \cos ^{2 q-1} \varphi \mathrm{d} \varphi
\end{gathered}
$$

Knowing that $h=y^{\prime}$, formula (21) demonstrates that on the equidistant parallel $\pm \varphi_{s}$, the $h=k=1$ identity holds regardless of the parameters $q$ and $\varphi_{B}$. This means that we may call the parallels $\pm \varphi_{s}$ of the best cylindrical map projections as standard parallels.

\section{Analysing the distortion of the best cylindrical map- ping for the whole Earth}

Until this point, the bounding latitude $\varphi_{B}$ of the spherical zone was arbitrary. In this section, $\varphi_{B}$ is assumed to be $90^{\circ}$, so the mapping of the whole Earth is considered. The limit of expression (20) in this case is:

$$
\begin{array}{r}
\ln \cos \varphi_{s}= \\
\lim _{\varphi_{B} \rightarrow \pi / 2}\left[\ln \cos \varphi_{B}+\frac{\ln \tan \left(\frac{\pi}{4}+\frac{\varphi_{B}}{2}\right)}{\sin \varphi_{B}}-1\right]= \\
\lim _{\varphi_{B} \rightarrow \pi / 2} \ln \left[\cos \varphi_{B} \tan \left(\frac{\pi}{4}+\frac{\varphi_{B}}{2}\right)\right]-1= \\
\lim _{\varphi_{B} \rightarrow \pi / 2} \ln \left[\frac{\cos \varphi_{B}}{\cos \left(\frac{\pi}{4}+\frac{\varphi_{B}}{2}\right)} \sin \left(\frac{\pi}{4}+\frac{\varphi_{B}}{2}\right)\right]-1= \\
\lim _{\varphi_{B} \rightarrow \pi / 2} \ln \left[\frac{\sin \varphi_{B}}{\frac{1}{2} \sin \left(\frac{\pi}{4}+\frac{\varphi_{B}}{2}\right)}\right]-1=\ln 2-1
\end{array}
$$

That is

$$
\varphi_{s}=\arccos \frac{2}{\mathrm{e}}
$$

On the other hand, the integrand in the expression (8) for the calculation of the overall distortion becomes indeterminate at the Poles. However, the limit of the integrand exists at $\varphi= \pm \pi / 2$ and may be calculated as (first only for the part containing areal distortion):

$$
\begin{gathered}
\lim _{\varphi \rightarrow \pm \pi / 2} q \ln ^{2} \frac{y^{\prime} \cos \varphi_{s}}{\cos \varphi} \cos \varphi= \\
\lim _{z \rightarrow 0} q \ln ^{2} \frac{\left(\frac{z}{\cos \varphi_{s}}\right)^{2 q-1} \cos \varphi_{s}}{z} z= \\
\lim _{z \rightarrow 0} q\left[(2 q-1)\left(\ln z-\ln \cos \varphi_{s}\right)+\ln \cos \varphi_{s}-\ln z\right]^{2} z= \\
q(2-2 q)^{2} \ln ^{2} \cos \varphi_{s} \lim _{z \rightarrow 0} z+q(2 q-2)^{2} \lim _{z \rightarrow 0} z \ln ^{2} z- \\
2 q(2-2 q)^{2} \ln \cos \varphi_{s} \lim _{z \rightarrow 0} z \ln z=0
\end{gathered}
$$

Similar reasoning may show that the other part of the integrand (the one containing angular distortion) also converges to zero, thus, the whole integrand is bounded. This means that the integrand is Riemann integrable, it is possible to calculate the overall distortion for the whole globe.

Analytic expression of $E^{2}$ in (8) is difficult but the following result was gained using the Wolfram Mathematica CAS:

$$
\begin{gathered}
E^{2}=q \ln ^{2}\left(\frac{2}{\mathrm{e}}\right)^{2-2 q}+2(q-1) q(\ln 4-2) \ln \left(\frac{2}{\mathrm{e}}\right)^{2-2 q}+ \\
\frac{1}{3}(q-1)\left[-3 \ln ^{2}\left(4^{q} \mathrm{e}^{-2 q}\right)+6 q(\ln 4-2) \ln \left(4^{q} \mathrm{e}^{-2 q}\right)+\right. \\
\left.q\left(\pi^{2}-3[8+(\ln 4-4) \ln 4]\right)\right]
\end{gathered}
$$

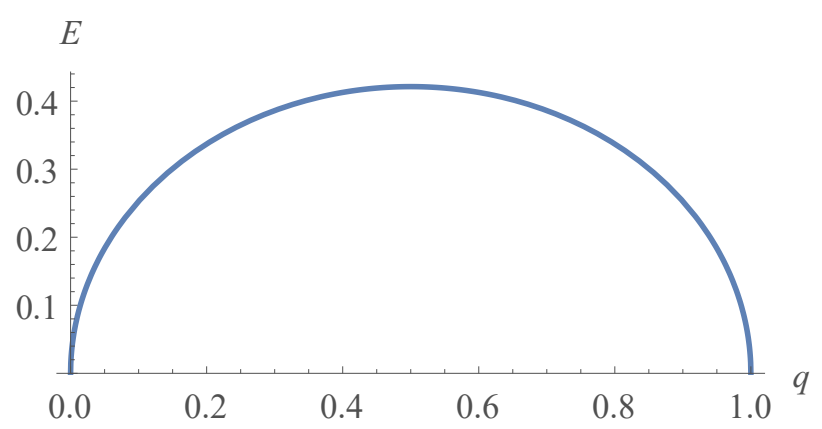

Figure 1. Overall distortion of the best cylindrical mapping according to the undesirability of areal distortion

This function is plotted on fig. 1. The reader is warned that a higher value of $E$ does not indicate larger distortion. This is because $E$ is calculated differently for each $q$. It should be understood as the "remaining trade-off" of the optimization. If one seeks an equal-area or conformal map 
without taking the other kind of distortion into account, no trade-off is necessary. The amount of this trade-off has its maximum at the balanced case $q=1 / 2$. The two extremal values at $q=0$ and $q=1$ show the evident phenomenon that areal and angular distortion can be perfectly eliminated separately. The plot is symmetrical around $q=1 / 2$. This suggest that areal and angular distortions are complementary of each other, they may be interchangeable in a pure mathematical sense. Although this plot is only valid within the family of cylindrical mappings, this behaviour might also be observed in other types of map projections. Further investigations are needed to confirm this.

The distortion values using various numbers for $q$ are not comparable to each other, as the formula for measuring the distortion includes this variable. To gain comparable results, the distortion value defined by eq. (7) was normalized against $q=1 / 2$, getting the original criterion of Kavrayskiy and Bayeva.

$$
\hat{E}^{2}=\frac{1}{S} \int_{S} \frac{\varepsilon_{p}^{2}+\varepsilon_{i}^{2}}{2} \mathrm{~d} S
$$

Symbolic calculation on the computer yielded the following distortion value for the best cylindrical map projection applied on the whole globe:

$$
\begin{aligned}
& \hat{E}^{2}= \frac{1}{6}\left[3 \ln ^{2}\left(\frac{2}{\mathrm{e}}\right)^{2-2 q}+6(q-1)(\ln 4-2) \ln \left(\frac{2}{\mathrm{e}}\right)^{2-2 q}-\right. \\
&(2[q-1] q+1)\left(\pi^{2}-3[8+(\ln 4-4) \ln 4]\right)+ \\
&\left.\quad 3 \ln \left(4^{q} \mathrm{e}^{-2 q}\right)\left(\ln \left[4^{q} \mathrm{e}^{-2 q}\right]-2 q[\ln 4-2]\right)\right]
\end{aligned}
$$

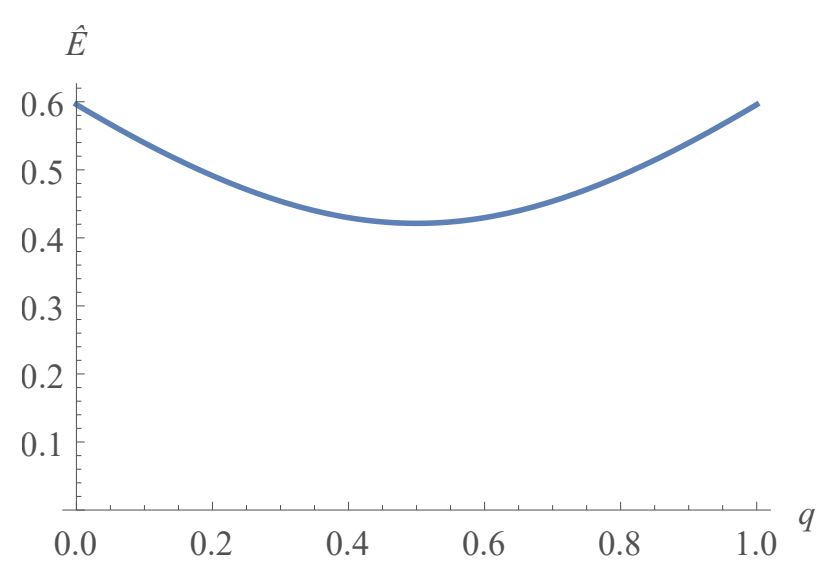

Figure 2. Overall distortion of the best cylindrical mapping according to the undesirability of areal distortion normalized for the Kavrayskiy's criterion

Plotting this function (fig. 2.) shows that this function is also symmetrical about $q=1 / 2$. However this is not evident: Györffy (1995) pointed out that similar symmetry is not present among conic and azimuthal map projections. It is obvious, that this function has its minimum at $q=1 / 2$ (as this best cylindrical map projection optimizes exactly this distortion value). Departing away from this balance, the distortion value increases slowly but a steep increase is observed before reaching the distortion values of the extremal conformal and equal-area maps. As the Airy-Kavrayskiy criterion very strongly correlates to the finite shape distortion of maps (Kerkovits, 2019), it can be concluded that different undesirability of areal and angular distortion does not influence the overall quality of the map projection significantly provided that the difference is not too big. One may recheck fig. 3. for a visual proof. Again, this can be rigorously stated only for cylindrical mappings.

\section{Conclusions}

The formula of the best cylindrical map projection was obtained for spherical zones even when the undesirability of areal and angular distortions were unequal. These mappings were found to be apt in traditional cartography. Furthermore, the overall distortion value of this projection was calculated for the whole globe analytically. The results suggest that areal and angular distortions have some kind of symmetrical and complementary nature.

\section{Acknowledgements}

The author thanks Györffy János for giving the idea for the present study and for his valuable comments on an earlier version of the manuscript.

\section{References}

Frančula, N., 1971. Die vorteilhaftesten Abbildungen in der Atlaskartographie. Doctoral dissertation, Reinischen Friedrich Wilhelms Universität, Hohen Landwirtschaftlichen Fakultät.

Grafarend, E. W. and Niermann, A., 1984. Beste echte zylinderabbildungen. Kartographische Nachrichten 34(3), pp. 103-107.

Györffy, J., 1990. Anmerkungen zur frage der besten echten zylinderabbildungen. Kartographische Nachrichten 40(4), pp. 140-146.

Györffy, J., 1995. Несколько свойств покозателей искажения картографических пройекций. Геодезия и аэрофботосъёмка (4), pp. 130-143.

Györffy, J., 2016. Some Remarks on the Question of Pseudocylindrical Projections with Minimum Distortions for World Maps. Springer International Publishing, Cham, pp. 253-265.

Kerkovits, K., 2019. Comparing finite and infinitesimal map distortion measures. International Journal of Cartography 5(1), pp. 3-22.

Kerkovits, K., 2020. A statistical reinterpretation and assessment of criteria used for measuring map projection distortion. Cartography and Geographic Information Science 47(6), pp. 481-491.

Lapaine, M. and Frančula, N., 2016. Map projection aspects. International Journal of Cartography 2(1), pp. 38-58.

Meshcheryakov, G. A., 1968. Teoreticheskie osnovy matematicheskoj kartografii. Nedra, Moscow.

Snyder, J. P., 1987. Map projections - A working manual. Vol. 1395, US Government Printing Office, Washington.

Баева [Bayeva], Е. Ю., 1987. Критерии оценки достоинства картографических проекций, исползуемых для составления карт Мира. Геодезия и аэробботосъёмка (3), pp. 109-112. 


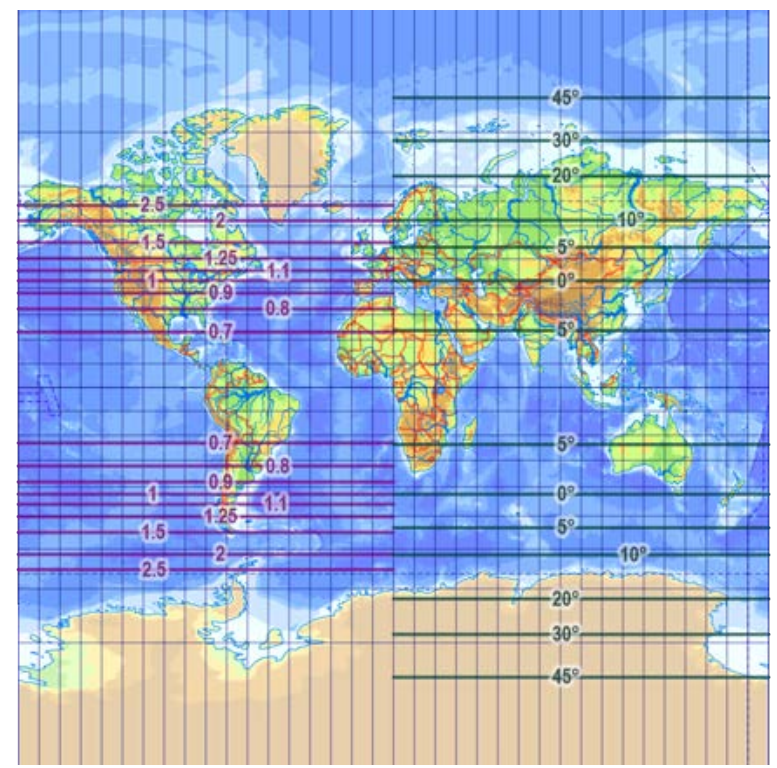

(a) $q=0.2$ (low angular distortion, polar regions clipped)

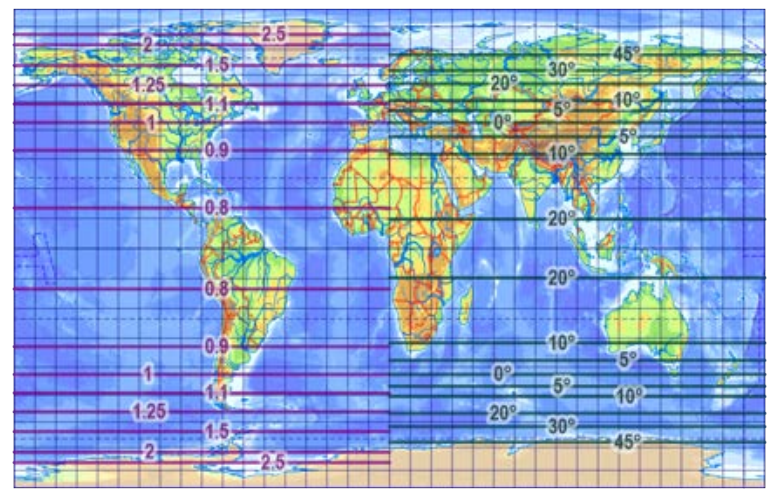

(c) $q=0.6$ (moderate areal distortion)

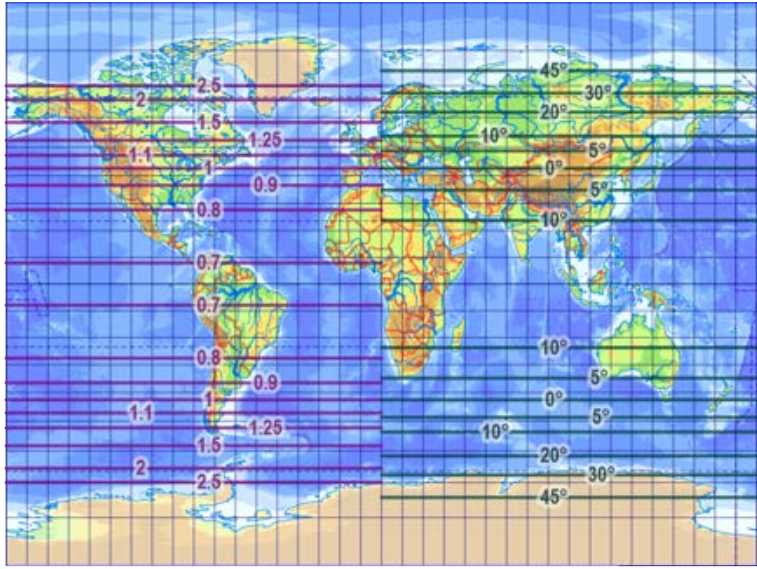

(b) $q=0.4$ (moderate angular distortion)

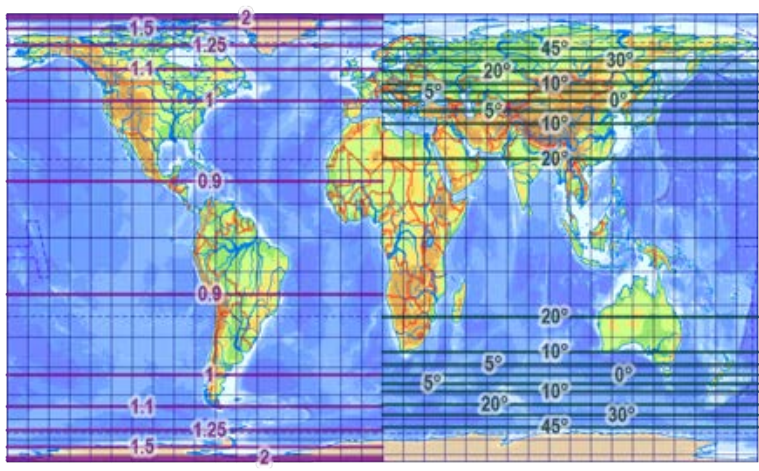

(d) $q=0.8$ (low areal distortion)

Figure 3. Areal distortion and maximal angular deviation of the best cylindrical projection $\left(\varphi_{B}=90^{\circ}\right)$ 